\title{
Epigenetic modulation of Wnt signaling contributes to neuropathic pain in rats
}

\author{
WEI FENG, RUYNAG TENG, YANG ZHAO, JIE GAO and HAICHEN CHU
}

\author{
Department of Anesthesiology, Affiliated Hospital of Qingdao University, Qingdao, Shandong 266021, P.R. China
}

Received August 24, 2014; Accepted May 15, 2015

DOI: $10.3892 / \mathrm{mmr} .2015 .3972$

\begin{abstract}
Previous studies have demonstrated that the Wnt/ $\beta$-catenin signaling pathway is critical to the induction and maintenance of chronic neuropathic pain caused by peripheral inflammation and nerve damage. Emerging evidence from recent studies suggests that epigenetic mechanisms may also be critical to the pathogenesis of chronic pain. The present study aimed to elucidate the epigenetic mechanisms underlying altered Wnt signaling and their involvement in CCI-induced neuropathic pain in rat sciatic nerves. The results of the present study demonstrated a significant increase in the expression levels of Wnt3a in the dorsal horn of the rats with CCI. In addition, a significant increase in histone $\mathrm{H} 3$ acetylation, and a significant decrease in cytosine methylation in the promoter region of Wnt3a was observed in the dorsal horn of the rats with CCI. Intrathecal application of XAV939, which acts as an inhibitor of Wnt signaling, significantly decreased the expression levels of active $\beta$-catenin, and attenuated the rat behavioral responses to thermal and mechanical pain stimuli. These results suggest that the epigenetic upregulation of Wnt3a in the dorsal horn contributes to the maintenance of pain-induced behavior in rats with CCI.
\end{abstract}

\section{Introduction}

Neuropathic pain is a common neurological disorder resulting from peripheral nerve damage, which affects millions of people worldwide (1). Wnt proteins are a family of secreted lipid-modified proteins, which act as signaling molecules in the regulation of cellular processes such as cell proliferation, differentiation, migration, and polarity, during the development of the cardiac and nervous systems $(2,3)$. To date, a total of 19 members of the human Wnt family have been identified. Wnt ligands bind to the cysteine-rich domain

Correspondence to: Dr Haichen Chu, Department of Anesthesiology, Affiliated Hospital of Qingdao University, 16 Jiangsu Road, Qingdao, Shandong 266021, P.R. China

E-mail: chuhaichen0312@126.com

Key words: neuropathic pain, Wnt, histone acetylation, cytosine methylation of frizzled receptors and co-receptors, in order to activate intracellular signaling cascades (2). Wnt signaling includes both canonical Wnt/ $\beta$-catenin pathways, and non-canonical $\beta$-catenin-independent pathways $(2,4-8)$. $\beta$-catenin is a multifunctional protein that interacts with transcription factors in order to activate the transcription of a target gene. Non-canonical $\beta$-catenin-independent pathways induce the release of intracellular calcium, which in turn activates a calcium-calmodulin-dependent kinase (CaMKII) $(4,5)$. Wnts are predominantly expressed in the brain, where they are critical to various neurological developmental processes $(2,3)$, as well as the regulation of synaptic plasticity $(3,5,9)$. Dysregulation of Wnt signaling is responsible for poor axonal regeneration, as well as the development of certain oncogenic processes and mental disorders (10-13). A recent study demonstrated the role of Wnt signaling in the development and maintenance of neuropathic pain in various rodent models (2). The present study aimed to investigate the functional significance of spinal Wnt signaling in a rodent model of neuropathic pain.

An increasing amount of evidence suggests that epigenetic modifications of DNA and/or histones in the transcriptional control region may significantly modulate the transcription of specific loci within the genome, thus critically influencing neuronal development, learning and memory, as well as various neurological disorders including chronic pain (3). Previous studies have reported that the suppression of histone acetylation via the enhanced activity of histone deacetylase 2, was may significantly decrease the expression levels of genes associated with synaptic plasticity, and modify cognitive function (3). A recent study also demonstrated the alteration of histone acetylation and cytosine methylation during the induction and maintenance of sustained pain-induced behavior, caused by peripheral inflammation and nerve damage (3). These epigenetic modifications present at specific genomic loci may significantly affect the expression levels of certain proteins, thus contributing to the sensitization of peripheral sensory neurons and central nociceptive neurons (5). The present study aimed to elucidate the epigenetic mechanisms underlying altered Wnt signaling in the rat spinal dorsal horn.

\section{Materials and methods}

Animals and CCI surgery. Adult male Wistar rats weighing 180-220 g were obtained from the Institutional Center of Experiment Animals (Quingdao, China), and were housed in 
standard laboratory conditions $\left(22 \pm 2^{\circ} \mathrm{C}\right.$ with a $12 \mathrm{~h}$ light/dark cycle). The rats were provided ad libitum access to food and water. The 88 rats were divided into four groups: control $(n=35)$, CCI $(n=35)$, CCI + XAV939 $(n=10)$ and control + XAV939 $(n=9)$. All animal experiments were approved by the Institutional Animal Care and Use Committee (Qingdao University, Qingdao, China), and conformed to the guidelines of the National Institution of Health (14).

For chronic constriction injury (CCI) surgery, all rats were anesthetized with $1 \%$ isoflurane. The right sciatic nerve was exposed and three Natural Chromic-Gut 4-0 absorbable sutures (Stoelting Co., Dublin, Ireland) were placed around the nerve with $1 \mathrm{~mm}$ between each suture. The muscle and skin layers were closed with silk sutures. In the rats of the sham (control) group, the sciatic nerve was exposed but no sutures were placed around the nerve.

The inhibitor of Wnt signaling XAV939, was purchased from Stemgent Inc. (Cambridge, MA, USA), and unless otherwise stated, all other chemicals and reagents were purchased from Sigma-Aldrich (St. Louis, MO, USA).

Behavioral tests. Intrathecal cannulation was performed as previously described (6). PE-10 polyethylene intrathecal catheters were implanted one week prior to the surgical procedures. The catheters were placed $8 \mathrm{~cm}$ into the caudal side of the rats through an incision in the cisternal membrane, and secured to the musculature at the incision site. Only the rats that showed no evidence of neurological deficits following catheter insertion were selected for further experimentation. A total of $5 \mu \mathrm{g}$ inhibitor of Wnt signaling XAV939 or vehicle (saline) in a $10 \mu 1$ total volume were administered daily into the rats, followed by a $10 \mu \mathrm{l}$ flush with normal saline.

Mechanical allodynia was detected using a series of von Frey filaments with logarithmic incremental stiffness (Stoelting Co., Wood Dale, IL, USA), and the 50\% probability of paw withdrawal thresholds were calculated using the up-down method, as previously described (7). The filaments were applied to the plantar surface of the hind paws for $\sim 6 \mathrm{sec}$ in an ascending or descending order following a negative or positive paw withdrawal response, respectively. Following the first observed change in behavioral response, the subsequent six consecutive responses were used to calculate the paw withdrawal pressure threshold (in grams). The maximum pressure threshold was set as $16 \mathrm{~g}$.

The thermal nociceptive thresholds were measured using radiant heat (8). Briefly, the rats were placed into individual plastic cubicles mounted on a glass surface maintained at $30^{\circ} \mathrm{C}$, and allowed a $1 \mathrm{~h}$ habituation period. A thermal stimulus from a radiant heat source was then delivered to the plantar surface of the hind paws. The time taken for the rat to remove the paw from the thermal stimulus was electronically recorded and determined as the paw withdrawal latency period. The stimulus ceased automatically when the hind paw moved, or following $16 \mathrm{sec}$, in order to prevent tissue damage. The intensity of the heat stimulus was maintained constant throughout all experiments.

Protein extraction and immunoblotting. Following behavioral testing at day 15 , the rats were anesthetized with $50 \mathrm{mg} / \mathrm{kg}$ pentobarbital sodium (P-010; Sigma-Aldrich, St. Louis, MO, USA), and transcardially perfused with $30 \mathrm{ml}$ phosphate-buffered saline. The ipsilateral dorsal horn tissue (L4-L6) was subsequently rapidly collected, and homogenized (Thermo Fisher Scientific, Waltham, MA, USA) in a lysis buffer supplemented with a protease inhibitor cocktail (1 $\mathrm{mM}$ phenylmethylsulfonyl fluoride, $1 \mathrm{mM} \mathrm{NaF}, 1 \mathrm{mM} \mathrm{NaVO}, 1 \mu \mathrm{g} / \mathrm{ml}$ leupeptin, $1 \mu \mathrm{g} / \mathrm{ml}$ pepstatin, and $1 \mu \mathrm{g} / \mathrm{ml}$ aprotinin). The lysates were centrifuged at $12,000 \mathrm{x} \mathrm{g}$ for $10 \mathrm{~min}$ at $4^{\circ} \mathrm{C}$, and the protein concentration was measured using the Bio-Rad Protein Assay kit (Bio-Rad Laboratories, Inc., Hercules, CA, USA). The samples were treated with SDS sample buffer at $95^{\circ} \mathrm{C}$ for $5 \mathrm{~min}$, separated by $10 \%$ SDS-PAGE, and blotted to a nitrocellulose membrane. The membranes were incubated overnight at $4{ }^{\circ} \mathrm{C}$ with the following primary antibodies: Anti-active $\beta$-catenin $(1: 1,000$; cat. no. 07-1651, EMD Millipore, Billerica, MA, USA), anti $\beta$-catenin (1:1,000; cat. no. 04-958, EMD Millipore), anti-Wnt3a (1:1,000; cat. no. ABD124, EMD Millipore), and anti-GAPDH (1:200; cat. no. sc-365062, Santa Cruz Biotechnology Inc., Dallas, TX, USA). The membranes were subsequently incubated with appropriate horseradish peroxidase-conjugated secondary antibodies against mouse IgG (1:10,000; cat. no. 715-035-150; Jackson ImmunoResearch, West Grove, PA, USA) or rabbit IgG (1:10,000, cat. no. 711-035-152, Jackson ImmunoResearch) at room temperature for $1 \mathrm{~h}$ and the blots were visualized using an enhanced chemiluminescence system (GE Healthcare Life Sciences, Little Chalfont, UK). The intensity of the bands were quantified using ImageJ analysis software (v1.48; National Institute of Health, Bethesda, MA, USA) and the ratios of the target proteins against GAPDH band density were calculated accordingly.

Chromatin immunoprecipitation (ChIP) and polymerase chain reaction $(P C R)$. A ChIP assay was performed as previously described (9). Briefly, the rat ipsilateral dorsal horn tissue samples (L4-L6) were collected, and fixed with $1 \%$ formaldehyde at $37^{\circ} \mathrm{C}$ for $5 \mathrm{~min}$. The chromatin fraction was extracted and fragmented to $400-500$ bp by sonication, as previously described (9). A total of $200 \mu \mathrm{l}$ sonicated chromatin in lysis buffer supplemented with $1 \%$ Triton $\mathrm{X}-100$ was pre-cleared using $5 \mu \mathrm{g}$ normal rabbit immunoglobulin $\mathrm{G}$ (IgG) conjugated to $30 \mu \mathrm{l}$ Protein $\mathrm{A}$, and subsequently incubated with either $5 \mu \mathrm{g}$ polyclonal acetylated histone H3 (EMD Millipore), or normal rabbit IgG conjugated to Protein $\mathrm{A}$ at $4^{\circ} \mathrm{C}$ for $20 \mathrm{~h}$. The immunoprecipitants were consecutively washed with $1 \mathrm{ml}$ of the following buffers: Low Salt Wash Buffer, High Salt Wash Buffer, LiCl Wash Buffer, and TE Buffer (10 mM Tris-HCl pH 8.0, 1 mM EDTA). Following reverse crosslinking at $65^{\circ} \mathrm{C}$ for $4 \mathrm{~h}$, the DNA was purified with ChIP DNA Clean \& Concentrator (Zymo Research, Irvine, CA, USA), and used as a template for the PCR, which was carried out using the SYBR Select PCR Master Mix (Applied Biosystems Life Technologies, Foster City, CA, USA) and the 7500 Fast Real-Time PCR system (Applied Biosystems Life Technologies). The PCR cycling conditions were as follows: Heating at $50^{\circ} \mathrm{C}$ for $2 \mathrm{~min}$ followed by $95^{\circ} \mathrm{C}$ for $10 \mathrm{~min}, 40$ cycles of $95^{\circ} \mathrm{C}$ for $15 \mathrm{sec}$, and $60^{\circ} \mathrm{C}$ for $1 \mathrm{~min}$. The following primers (SBS Genetech Co., Ltd., Beijing, China) were designed to amplify $\sim 200 \mathrm{bp}$ fragments in the promoter region of the target genes: Wnt3a forward, 5'-GAGCTCTGCCATTCACCCTA-3', and reverse, 5'-GGCTGCAGAGCACTAAGAGG-3'; and GAPDH forward, 5'-AGACAGCCGCATCTTCTTGT-3', and reverse 
5'-CGTCCTCTACCATCCTCTGC-3'. The ChIP signal was calculated according to the manufacturer's instructions, using the following method (15): $\Delta \mathrm{Ct}_{\text {[normalized ChIP] }}=\left(\mathrm{Ct}_{[\mathrm{ChIP]}}-\left(\mathrm{Ct}_{[\text {[Input] }}\right.\right.$ - $\log _{2}$ (input dilution factor))); and the ChIP/Input ratio was calculated as $2^{(-\Delta \mathrm{C} \text { tnormalized ChIP) }}$.

Methylated DNA immunoprecipitation (MeDIP) Assay. The MeDIP assay was performed as previously described with minor modifications (10). Briefly, the rat ipsilateral dorsal horn tissue samples (L4-L6) were collected, and the genomic DNA was fragmented into $400-500 \mathrm{bp}$ as described above. The sonicated samples were centrifuged at $14,000 \mathrm{x}$ g for $10 \mathrm{~min}$, and the supernatants were subsequently collected. The samples were then incubated with a polyclonal antibody targeting 5-methylcytosine (1:100, EMD Millipore) overnight at $4^{\circ} \mathrm{C}$ with gentle agitation. The DNA-antibody complex was enriched with Protein A agarose beads, and the DNA fragments in the input and pulled-down fractions were purified. A PCR was performed to amplify the $\sim 200 \mathrm{bp}$ segments corresponding to the $\mathrm{CpG}$ sites within the Wnt3a promoter region. The following primers (SBS Genetech Co., Ltd.) were used to amplify the fragments: Wnt3a forward, 5'-GAGCTCTGCCATTCACCCTA-3', and reverse, 5'-GGCTGCAGAGCACTAAGAGG-3'; and GAPDH forward, 5'-AGACAGCCGCATCTTCTTGT-3', and reverse, 5'-CTGCGGGAGAAGAAAGTCAG-3'. All amplifications were carried out in triplicate, and the PCR data were analyzed as described above.

Reverse transcription-quantitative PCR (RT-qPCR). The ipsilateral dorsal horn tissues (L4-L6) were collected from the rats. Total RNA was extracted using TRIzol ${ }^{\circledR}$ reagent (Invitrogen Life Technologies, Carlsbad, CA, USA), and reverse transcribed using SuperScript III Reverse Transcriptase (Invitrogen Life Technologies). The following primers (SBS Genetech Co., Ltd.) were designed to amplify the $200 \mathrm{bp}$ fragment within the cDNA sequence of the target genes: Wnt3a forward, 5'-ATGGCTCCTCTCGGATACCT-3', and reverse, 5'-GGGCATGATCTCCACGTAGT-3'; and GAPDH forward, 5'-AGACAGCCGCATCTTCTTGT-3', and reverse 5'-CTTGCCGTGGGTAGAGTCAT-3'. RT-qPCR was performed using SYBR Green qPCR SuperMix (Invitrogen Life Technologies). The cycle threshold $(\mathrm{Ct})$ values for each sample were determined with logarithmic phase amplification plots. The data were subsequently analyzed using the $2^{-\Delta \Delta \mathrm{Ct}}$ method.

Bisulfite sequencing PCR. The ipsilateral dorsal horn tissue samples (L4-L6) were collected from the rats. Total DNA was subsequently extracted, fragmented to $400-500 \mathrm{bp}$, and purified. The bisulfite conversion of the DNA was performed using the EZ DNA Methylation-Gold ${ }^{\mathrm{TM}}$ kit (Zymo Research) as previously described (11). A $250 \mathrm{bp}$ fragment in the promoter region of Wnt3a was amplified, independent of its methylation status, using the following primers (SBS Genetech Co., Ltd.): Forward, 5'-GGGTTTTAGTGTTATTTTAGGGTAAT-3', and reverse, 5'-ACATAAACCTTCACTACCCTCTTTC-3'. The PCR product was then purified using a Gel Extraction kit (Qiagen, Inc., Valencia, CA, USA), and sequenced using a reverse primer (Qiagen). The methylation percentage of each $\mathrm{CpG}$ site within the amplified promoter region of Wnt3a was determined by calculating the ratio between the guanine $(\mathrm{G})$ and adenine (A) peaks $[\mathrm{G} /(\mathrm{G}+\mathrm{A})]$, and these electropherogram ratios were subsequently analysed using Chromas software (v.2.1.1; Technelysium Pty, Ltd., South Brisbane, Australia).

Statistical analysis. All data were presented as the mean \pm standard error of the mean, and were analyzed using a Student's t-test or two-way analysis of variance, followed by Fisher's least significant difference post hoc analysis. Statistical analysis was performed using StatView 5.0 software (Cary, NC, USA). $\mathrm{P}<0.05$ was considered to indicate a statistically significant difference.

\section{Results}

Increased Wnt signaling in the dorsal horn of the rats with CCI. The expression levels of Wnt3a were significantly increased in the dorsal horn (L4-L6) of the rats with CCI, as compared with the rats in the sham group (Fig. $1 \mathrm{~A}, \mathrm{P}<0.01, \mathrm{n}=7$ ). These results were concordant with the observed increase in mRNA expression levels of Wnt3a in the dorsal horn of the rats with CCI (Fig. 1B, $\mathrm{P}<0.01, \mathrm{n}=7$ ). The results of the present study also showed that the expression levels of active $\beta$-catenin, the downstream effector of Wnt signaling, were significantly increased in the dorsal horn (L4-L6) of the rats with CCI (Fig. 2, $\mathrm{P}<0.01, \mathrm{n}=7$ ). These results demonstrate the presence of increased Wnt signaling in the dorsal horn of rats with CCI.

Epigenetic modifications of the Wnt3a promoter region. Previous studies have demonstrated that the epigenetic modification of histone acetylation and/or cytosine methylation may significantly influence chromatin remodeling, thus regulating the transcription of target genes $(4,10)$. The present study examined the extent of histone $\mathrm{H} 3$ acetylation and cytosine methylation in the promoter region of Wnt3a, in the lumbar dorsal horn of rats with CCI. The ChIP assay used polyclonal antibodies targeting acetylated histone $\mathrm{H} 3$ and demonstrated that the acetylation of histone $\mathrm{H} 3$ was significantly increased in the promoter region of Wnt3a, but not GAPDH, in the lumbar dorsal horn of the rats with CCI (Fig. 3; $\mathrm{P}<0.01, \mathrm{n}=7$ ). Conversely, the results showed that when using the polyclonal antibodies targeting 5'-cytosine, cytosine methylation was significantly decreased in the promoter region of Wnt3a, but not GAPDH, in the lumbar dorsal horn of the rats with $\mathrm{CCI}$ (Fig. 4A; $\mathrm{P}<0.01 ; \mathrm{n}=7$ ). Furthermore, the results of the bisulfite sequencing PCR demonstrated the alteration of methylation at specific CpG sites in the Wnt3a promoter region in the lumbar dorsal horn of the rats with CCI (Fig. 4B, n=6). These results demonstrate the presence of substantial epigenetic modification within the Wnt3a promoter region, which may contribute to the upregulation of Wnt3a signaling in the lumbar dorsal horn of rats with CCI.

Inhibition of Wnt3a signaling attenuates the pain-induced behaviors of rats with CCI. In order to investigate the functional significance of increased Wnt3a expression in the pain-induced behavior of the rats with CCI, $5 \mu \mathrm{g}$ of either XAV939 (Wnt3a inhibitor), or vehicle were administered to the rats with CCI via an intrathecal catheter. Treatment with XAV939 significantly attenuated the expression levels of 
A
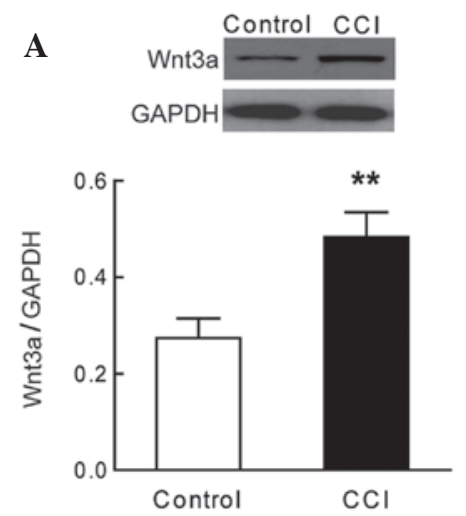

B

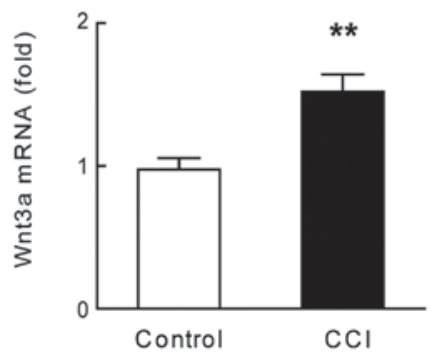

Figure 1. Increased expression levels of Wnt3a in the dorsal horn of the rats with chronic constriction injury (CCI). Following CCI, a significant increase in the (A) protein and (B) mRNA expression levels of Wnt3a were observed in the dorsal horn of the rats with CCI, as compared with the control group (n=7). The data are expressed as the mean \pm standard deviation ( ${ }^{* *} \mathrm{P}<0.01$, vs. the control group).

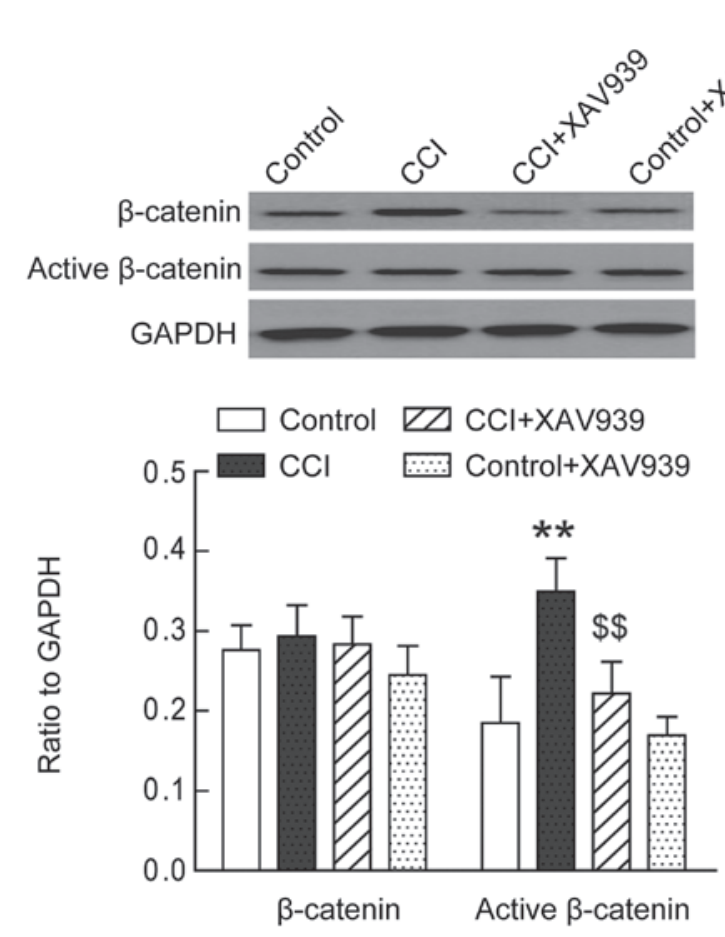

Figure 2. Inhibition of Wnt3a signaling by treatment with $5 \mu \mathrm{g}$ XAV939 significantly decreased the expression levels of active $\beta$-catenin in the lumbar dorsal horn of the rats with chronic constriction injury (CCI), as compared with the control group $(n=7)$. The data are expressed as the mean \pm standard deviation $\left({ }^{* *} \mathrm{P}<0.01\right.$, vs. the control group: ${ }^{\$} \mathrm{P}<0.01$, vs the $\mathrm{CCI}$ group).

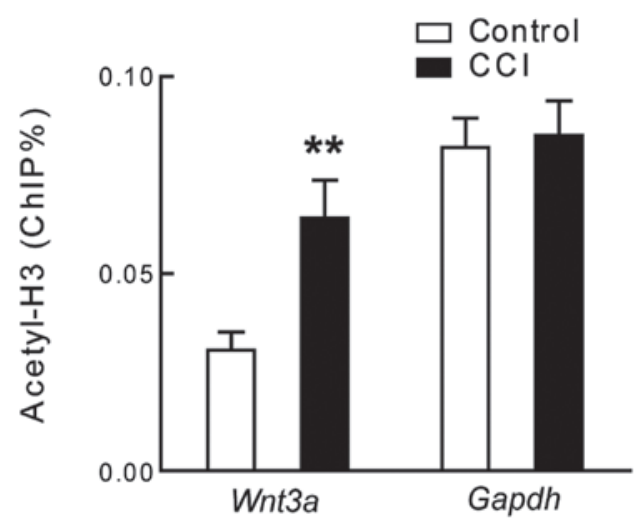

Figure 3. Increase in histone $\mathrm{H} 3$ acetylation in the promoter region of Wnt3a, but not GAPDH, in the dorsal horn tissue samples of the rats with CCI, as compared with the control group $(\mathrm{n}=7)$. The data are expressed as the mean \pm standard deviation ( ${ }^{* *} \mathrm{P}<0.01$, vs. the control group). ChIP, chromatin immunoprecipitation. 
A

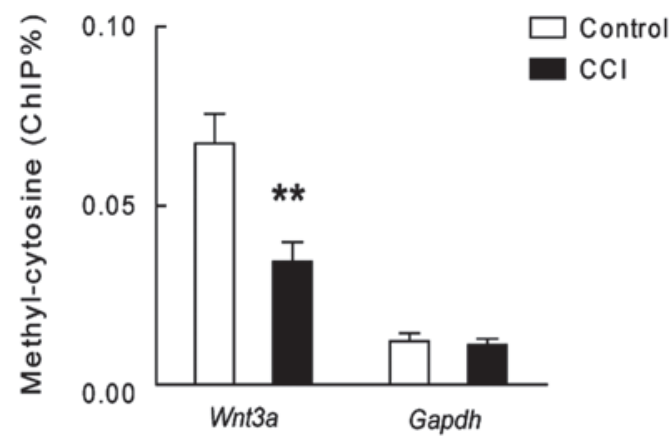

CCGATTTGGTGGTGAGTGAGTCTCCTTGCGTTCGCCCCC $\overline{\text { GGGCCCCAGTGCCACCCCAGG }}$

$\overrightarrow{\text { GCAAC }^{1} \text { CGCCCCTCCTGGGGCAGGGCCCCCAC }}{ }^{2} \mathrm{CGT}^{3} \mathrm{CGCTCTG}^{4} \mathrm{CGCACTGGCTG}^{5} \mathrm{CGGG}^{6} \mathrm{CGGG}$

TTGTTTTCCTTGGCTGCCACTTGGACCTGTT ${ }^{7} \mathrm{CGGGC}^{8} \mathrm{CGCAGCTGGGA}^{9} \mathrm{CG}^{10} \mathrm{CGGGTGG}^{11} \mathrm{CGGA}$

GCTTC $^{12}$ CGGGGACACTGATTCCCCTGGCAGGAGGGGAAAGAGGGCAGTGAAGGTCCATGCT

B

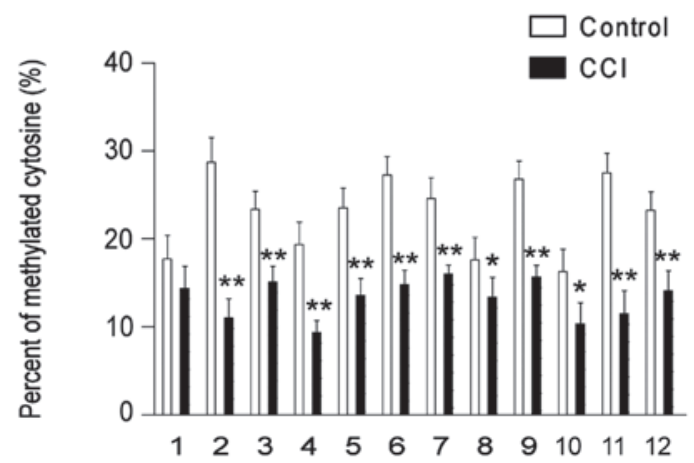

Figure 4. Decrease in cytosine methylation in the promoter region of Wnt3a in the dorsal horn tissue of the rats with chronic constriction injury (CCI). (A) Chromatin immunoprecipitation (ChIP) assay with polyclonal antibodies targeting 5'-methylcytosine showed a decreased in cytosine methylation in the promoter region of Wnt3a, but not GAPDH, as compared with the control group ( $\mathrm{n}=7$ ). (B) Methylation of specific CpG sites within the promoter region of Wnt3a in the dorsal horn of rats with CCI $(\mathrm{n}=6)$. The data are expressed as the mean \pm standard deviation $\left(" \mathrm{P}<0.05\right.$ and ${ }^{* *} \mathrm{P}<0.01$, vs. the control group).
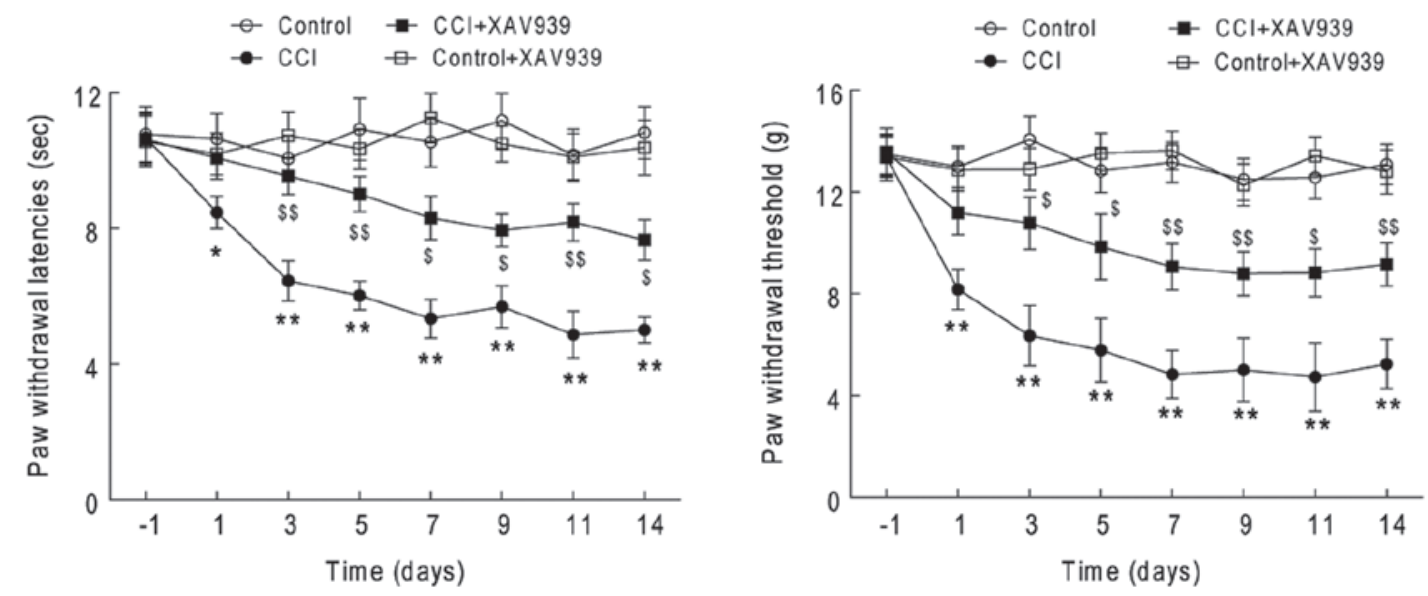

Figure 5. Inhibition of Wnt3a signaling by $5 \mu \mathrm{g}$ XAV939 attenuated the behavioral responses to mechanical and radiant thermal stimuli in the rats with chronic constriction injury $(\mathrm{CCI})(\mathrm{n}=10)$. The data are expressed as the mean \pm standard deviation $\left({ }^{*} \mathrm{P}<0.05\right.$ and ${ }^{* *} \mathrm{P}<0.01$, vs. the control group: ${ }^{\mathrm{S}} \mathrm{P}<0.05$ and ${ }^{\$ \$} \mathrm{P}<0.01$, compared with the CCI group).

active $\beta$-catenin in the lumbar dorsal horn of the rats with CCI (Fig. 2; $\mathrm{P}<0.01 ; \mathrm{n}=7$ ), whereas no change in the expression levels of active $\beta$-catenin was observed in the sham group rats. The behavioral studies also showed that treatment with
XAV939 significantly attenuated the behavioral responses to mechanical and radiant thermal stimuli of the rats with CCI (Fig. 5, $\mathrm{P}<0.01, \mathrm{n}=10$ ), whereas it did not significantly change the behavioral responses of the rats in the sham (control) group. 
These results indicate that the upregulation of spinal Wnt3a signaling may significantly contribute to the pain-induced behavior of the rats with CCI.

\section{Discussion}

Wnt signaling was originally thought to be exclusively involved in the regulation of cellular processes such as proliferation, differentiation, and migration during neuronal development; however, recent studies have demonstrated the important role of Wnt signaling in the pathogenesis of neuropathic and bone cancer-induced pain $(2,12)$. A previous study reported that treatment with Wnt3a significantly enhanced A $\delta$ fiber response to mechanical stimuli, and induced murine hypersensitivity to mechanical and thermal stimuli (13). Nerve injury and bone cancer cause rapid and long-lasting upregulation of Wnts, such as Wnt3a, as well as activation of the Wnt-Frizzled- $\beta$-catenin signaling pathway in primary sensory neurons and spinal dorsal horn nociceptive neurons, which contribute to the development and maintenance of thermal hyperalgesia and mechanical allodynia. These responses may be substantially attenuated by the spinal injection of inhibitors of Wnt signaling $(2,16)$. A recent study also showed that partial sciatic nerve ligation induced the upregulation of spinal Wnt3a expression, and the inhibition of Wnt/ $\beta$-catenin signaling, which attenuated the induction of neuropathic pain (17). Activation of Wnt signaling may contribute to synaptic plasticity in dorsal horn neurons, and may be involved in the pathogenesis of human immunodeficiency virus-associated chronic pain (18). The present study detected increased expression levels of Wnt3a in the ipsilateral dorsal horn of a rodent model of CCI, and demonstrated that inhibition of Wnt signaling significantly attenuated the rat behavioral responses to mechanical and radiant thermal stimuli. These results further confirmed the involvement of Wnt3a signaling in the pathogenesis of CCI-induced neuropathic pain.

A previous study showed that epigenetic mechanisms were critically involved in the development and maintenance of persisted pain-induced behavior in peripheral inflammation and nerve injury (3). Peripheral inflammation also reportedly induced significant hypermethylation of $\mathrm{CpG}$ islands in the miR-219 promoter, which in turn led to the upregulation of spinal CaMKII $\gamma$, and the increase in pain-induced behavior of rats injected with complete Freud's adjuvant (19). Persistent peripheral inflammation has been shown to upregulate the expression of transcriptional regulator $\mathrm{MeCP} 2$ in the central nucleus of the mouse amygdala, which consequently resulted in repression of the expression of histone dimethyltransferase G9a, which resulted in increased central expression of brain-derived neurotrophic factor, thus inducing mouse hypersensitivity to painful stimuli (20). A previous study also reported that the epigenetic suppression of GAD65 via histone hypoacetylation in the brain stem neurons had a critical role in the induction and maintenance of pain-induced behavior caused by peripheral inflammation and nerve injury (21). The present study demonstrated the presence of increased expression levels of spinal Wnt3a due to significant epigenetic modifications in the promoter region of Wnt $3 \mathrm{a}$, which contributed to the pain-induced behavior of rats with CCI. These results, together with the findings of previous studies, strongly suggest that the epigenetic modification of specific genes may substantially contribute to the sensitization of peripheral and central nociceptive circuits, and to the induction and maintenance of pain-induced behavior in a rodent pain model.

The addition of a methyl group to the 5 position of cytosine in the $\mathrm{CpG}$ sites of a promoter region, catalyzed by DNA methyltransferases, may lead to transcriptional silencing via the recruitment of transcriptional repressors, and to chromatin condensation, thus restricting the access of transcriptional proteins, and masking the binding sites of transcriptional activators (22). Conversely, histone lysine acetylation has been associated with active transcription of target genes, whereas deacetylated histones were mostly detected within transcriptionally silenced domains (23). The alteration of histone acetylation and/or cytosine methylation in the transcriptional control region of specific genes has been extensively investigated in numerous neurological disorders (24), including neuropathic pain (5). Increased cytosine methylation of the transcriptional control region of the gene encoding endothelin-1 B receptor in sensory neurons has previously been shown to be associated with pain-induced behavior in a murine model of oral squamous cell carcinoma (25). In addition, the expression levels of lysine 9-acetylated histone $\mathrm{H} 3$ in the promoter regions of the chemokine CCL2 and CCL3 genes have been shown to be increased in injured sciatic nerves, which was associated with pain-induced behavior (26). The present study demonstrated an increase in the expression levels of spinal Wnt3a caused by increased histone H3 acetylation, and decreased cytosine methylation in the Wnt3a promoter region of rats with $\mathrm{CCI}$, which may contribute to the development and maintenance of pain-induced behaviors in a rodent model of neuropathic pain.

In conclusion, the present study demonstrated an increase in Wnt3a-mediated signaling, resulting from increased histone acetylation, and decreased cytosine methylation in the promoter region of Wnt $3 \mathrm{a}$, in the dorsal horn of rats with CCI. In addition, the results of the present study showed that the inhibition of Wnt3a signaling was able to significantly attenuate the pain-induced behavior of rats with CCI. Therefore, these results provide a novel insight into the development of therapeutic strategies for the treatment of neuropathic pain.

\section{References}

1. Gilron I, Baron R and Jensen T: Neuropathic pain: Principles of diagnosis and treatment. Mayo Clin Proc 90: 532-545, 2015.

2. Zhang YK, Huang ZJ, Liu S, Liu YP, Song AA and Song XJ: WNT signaling underlies the pathogenesis of neuropathic pain in rodents. J Clin Invest 123: 2268-2286, 2013.

3. Denk F and McMahon SB: Chronic pain: Emerging evidence for the involvement of epigenetics. Neuron 73: 435-444, 2012.

4. Guan JS, Haggarty SJ, Giacometti E, Dannenberg JH, Joseph N, Gao J, Nieland TJ, Zhou Y, Wang X, Mazitschek R, et al: HDAC2 negatively regulates memory formation and synaptic plasticity. Nature 459: 55-60, 2009.

5. Denk F, McMahon SB and Tracey I: Pain vulnerability: A neurobiological perspective. Nat Neurosci 17: 192-200, 2014.

6. Zhou HY, Chen SR, Chen H and Pan HL: The glutamatergic nature of TRPV1-expressing neurons in the spinal dorsal horn. J Neurochem 108: 305-318, 2009.

7. Zhang J, Wu D, Xie C, Wang H, Wang W, Zhang H, Liu R, Xu LX and Mei XP: Tramadol and propentofylline coadministration exerted synergistic effects on rat spinal nerve ligation-induced neuropathic pain. PLoS One 8: e72943, 2013. 
8. Hargreaves K, Dubner R, Brown F, Flores C and Joris J: A new and sensitive method for measuring thermal nociception in cutaneous hyperalgesia. Pain 32: 77-88, 1988.

9. Fujita T, Ryser S, Tortola S, Piuz I and Schlegel W: Gene-specific recruitment of positive and negative elongation factors during stimulated transcription of the MKP-1 gene in neuroendocrine cells. Nucleic Acids Res 35: 1007-1017, 2007.

10. Provencal N, Suderman MJ, Caramaschi D, Wang D, Hallett M, Vitaro F, Tremblay RE and Szyf M: Differential DNA methylation regions in cytokine and transcription factor genomic loci associate with childhood physical aggression. PLoS One 8: e71691, 2013.

11. Hayatsu H, Tsuji K and Negishi K: Does urea promote the bisulfite-mediated deamination of cytosine in DNA? Investigation aiming at speeding-up the procedure for DNA methylation analysis. Nucleic Acids Symp Ser (Oxf) 50: 69-70, 2006

12. Ji RR, Xu ZZ and Gao YJ: Emerging targets in neuroinflammation-driven chronic pain. Nat Rev Drug Discov 13: 533-548, 2014.

13. Simonetti M, Agarwal N, Stösser S, Bali KK, Karaulanov E, Kamble R, Pospisilova B, Kurejova M, Birchmeier W, Niehrs C, et al: Wnt-Fzd signaling sensitizes peripheral sensory neurons via distinct noncanonical pathways. Neuron 83: 104-121, 2014.

14. Guide for the care and use of laboratory animals. 8th Edition. Committee for the update of the guide for the care and use of laboratory animals. National Research Council of the National Academies. National Academies Press. 2013. Washongton, D.C.

15. Livak KJ and Schmittgen TD: Analysis of relative gene expression data using real-time quantitative PCR and the 2(-Delta Delta C(T)) Method. Methods 25: 402-408, 2001.

16. Tang SJ: Synaptic activity-regulated Wnt signaling in synaptic plasticity, glial function and chronic pain. CNS Neurol Disord Drug Targets 13: 737-744, 2014.
17. Itokazu T, Hayano Y, Takahashi R and Yamashita T: Involvement of Wnt/ $\beta$-catenin signaling in the development of neuropathic pain. Neurosci Res 79: 34-40, 2014.

18. Shi Y, Shu J, Gelman BB, Lisinicchia JG and Tang SJ: Wnt signaling in the pathogenesis of human HIV-associated pain syndromes. J Neuroimmune Pharmacol 8: 956-964, 2013.

19. Pan Z, Zhu LJ, Li YQ, Hao LY, Yin C, Yang JX, Guo Y, Zhang S, Hua L, Xue ZY, et al: Epigenetic modification of spinal miR-219 expression regulates chronic inflammation pain by targeting CaMKII $\gamma$. J Neurosci 34: 9476-9483, 2014.

20. Zhang Z, Tao W, Hou YY, Wang W, Kenny PJ and Pan ZZ: $\mathrm{MeCP} 2$ repression of $\mathrm{G} 9 \mathrm{a}$ in regulation of pain and morphine reward. J Neurosci 34: 9076-9087, 2014

21. Zhang Z, Cai YQ, Zou F, Bie B and Pan ZZ: Epigenetic suppression of GAD65 expression mediates persistent pain. Nat Med 17: 1448-1455, 2011

22. Cheng X: Structural and functional coordination of DNA and histone methylation. Cold Spring Harb Perspect Biol 6: a018747, 2014.

23. Sultan FA and Day JJ: Epigenetic mechanisms in memory and synaptic function. Epigenomics 3: 157-181, 2011.

24. Klein CJ and Benarroch EE: Epigenetic regulation: basic concepts and relevance to neurologic disease. Neurology 82: 1833-1840, 2014

25. Viet CT, Ye Y, Dang D, Lam DK, Achdjian S, Zhang J and Schmidt BL: Re-expression of the methylated EDNRB gene in oral squamous cell carcinoma attenuates cancer-induced pain. Pain 152: 2323-2332, 2011.

26. Kiguchi N, Kobayashi Y, Saika F and Kishioka S: Epigenetic upregulation of CCL2 and CCL3 via histone modifications in infiltrating macrophages after peripheral nerve injury. Cytokine 64: 666-672, 2013. 